\title{
Comparison of alginate and pectin based beads for production of poultry probiotic cells.
}

\begin{abstract}
A comparative study on the stability and potential of alginate and pectin based beads for production of poultry probiotic cells using MRS medium in repeated batch fermentation was conducted. The bead cores, made of three types of materials, i.e., ca-alginate, ca-pectinate and ca-alginate/pectinate, were compared. The effect of single and double layer coatings using chitosan and core material, respectively, on the bead stability and cell production were also studied. The pectin based beads were found to be more stable than that of the alginate beads and their stability was further improved by coating with chitosan. The cell concentration in pectin based beads was comparable to that in the alginate beads. On the other hand, pectin based beads gave significantly lower cell concentration in the growth medium for the initial fermentation cycles when compared to the alginate beads. In conclusion, pectin was found to be potential encapsulation material for probiotic cell production owing to its stability and favourable microenvironment for cell growth.
\end{abstract}

Keyword: Encapsulation; Pectin; Alginate; Bead; Probiotic; Stability 